\title{
BMJ Open Determinants of cigarette smoking status in a national cohort of black and white adult ever smokers in the USA: a cross-sectional analysis of the REGARDS study
}

\author{
Trisha M Parekh, ${ }^{\oplus 1}$ Chengyi Wu, ${ }^{2}$ Leslie A McClure, ${ }^{2}$ Virginia J Howard, ${ }^{3}$ \\ Mary Cushman, ${ }^{4}$ Angela M Malek, ${ }^{5}$ Kathleen F Harrington, ${ }^{1}$ Andrea L Cherrington, ${ }^{6}$ \\ Mark T Dransfield, ${ }^{1}$ Smita Bhatia ${ }^{7}$
}

To cite: Parekh TM, Wu C, McClure LA, et al. Determinants of cigarette smoking status in a national cohort of black and white adult ever smokers in the USA: a crosssectional analysis of the REGARDS study. BMJ Open 2019;9:e027175. doi:10.1136/ bmjopen-2018-027175

- Prepublication history and additional material for this paper are available online. To view these files, please visit the journal online (http://dx.doi org/10.1136/bmjopen-2018027175).

Received 10 0ctober 2018 Revised 4 April 2019 Accepted 5 April 2019

Check for updates

(C) Author(s) (or their employer(s)) 2019. Re-use permitted under CC BY-NC. No commercial re-use. See rights and permissions. Published by BMJ.

For numbered affiliations see end of article.

Correspondence to Dr Trisha M Parekh; trisha.parekh@gmail.com

\section{ABSTRACT}

Objectives While awareness of cigarette smoking's harmful effects has increased, determinants associated with smoking status remain understudied, including potential racial differences. We aim to examine factors associated with former versus current smoking status and assess whether these associations differed by race. Setting We performed a cross-sectional analysis using the population-based Reasons for Geographic and Racial Differences in Stroke(REGARDS)study.

Outcome measures Logistic regression was used to calculate the $\mathrm{OR}$ of former smoking status compared with current smoking status with risk factors of interest. Race interactions were tested using multiplicative interaction terms.

Results 16463 participants reported smoking at least 100 cigarettes in their lifetime. Seventy-three per cent $(n=12067)$ self-reported former-smoker status. Physical activity (reference (REF) $<3 \times /$ week; $>3 \times /$ week: $\mathrm{OR}=1.26$, $95 \% \mathrm{Cl} 1.11$ to 1.43 ), adherence to Mediterranean diet (REF: low; medium: $\mathrm{OR}=1.46,95 \% \mathrm{Cl} 1.27$ to 1.67; high: $\mathrm{OR}=2.20,95 \% \mathrm{Cl} 1.84$ to 2.64 ), daily television viewing time (REF: $>4$ hours; $<1$ hour: $\mathrm{OR}=1.32,95 \% \mathrm{Cl} 1.10$ to 1.60$)$ and abstinence from alcohol use (REF: heavy; none: $\mathrm{OR}=1.50,95 \% \mathrm{Cl} 1.18$ to 1.91 ) were associated with former-smoker status. Male sex, higher education and income $\$ 35000-\$ 74000$ (REF: $<\$ 20000$ ) were also associated with former-smoker status. Factors associated with lower odds of reporting former-smoker status were younger age (REF: $\geq 65$ years; $45-64$ years: $0 \mathrm{R}=0.34$, $95 \% \mathrm{Cl} 0.29$ to 0.39 ), black race (OR=0.62, $95 \% \mathrm{Cl} 0.53$ to 0.72 ) and single marital status (REF: married status; $\mathrm{OR}=0.66,95 \% \mathrm{Cl} 0.51$ to 0.87$)$, being divorced $(\mathrm{OR}=0.60$, $95 \% \mathrm{Cl} 0.50$ to 0.72 ) or widowed $(\mathrm{OR}=0.70,95 \% \mathrm{Cl} 0.57$ to 0.85$)$. Significant interactions were observed between race and alcohol use and dyslipidaemia, such that black participants had higher odds of reporting former-smoker status if they were abstinent from alcohol $(0 R=2.32$, $95 \% \mathrm{Cl} 1.47$ to 3.68 ) or had a history of dyslipidaemia $(0 \mathrm{R}=1.31,95 \% \mathrm{Cl} 1.06$ to 1.62$)$, whereas these relationships were not statistically significant in white participants.

\section{Strengths and limitations of this study}

- Using a cohort that oversampled black smokers and those living in the southeastern USA, we analysed the associations of potentially modifiable social and behavioural determinants of smoking status in a large cohort of black and white smokers.

- To further investigate tobacco-related differences in smoking status, we analysed the independent interactions of demographic, social, psychosocial, economic and behavioural factors with race.

- Our study is a cross-sectional analysis, and therefore the data presented do not establish causation.

- Covariates included in our analyses were assessed at the time of study entry and not necessarily at the time of change in smoking status.

- Factors associated with smoking status may represent co-occurring changes rather than predictive determinants.

Conclusion Efforts to promote tobacco cessation should consist of targeted behavioural interventions that incorporate racial differences.

\section{INTRODUCTION}

Despite the fact that tobacco use is on a decline in the USA, $\sim 15 \%$ of Americans continue to smoke, ${ }^{1}$ contributing to significant morbidity and mortality, including heart disease, stroke, chronic obstructive pulmonary disease and lung cancer. ${ }^{2}$ In the past 50 years, 20 million premature deaths have been attributed to smoking and secondhand smoke exposure, ${ }^{3}$ with tobacco-related morbidity and mortality disproportionately affecting racial minorities and individuals in low socioeconomic groups. ${ }^{45}$ Disparities in tobacco-related morbidity and mortality are poorly understood; however, individual and 
environmental characteristics have been considered. Tobacco use, primarily in the form of cigarettes, is most prevalent in the midwestern and southern regions of the USA, among those with disabilities and those with lower socioeconomic status. ${ }^{16}$ Black smokers start smoking later in life and smoke fewer cigarettes than white smokers, yet they inhale $30 \%$ more nicotine per cigarette. ${ }^{7}$ They are less likely to have access to health insurance ${ }^{8}$ or to use pharmacological aids to help quit smoking. ${ }^{9}$ Point-of-sale marketing of menthol cigarettes is targeted to communities with predominantly black residents ${ }^{10}$ who are known prominent consumers. ${ }^{11}$ Despite starting to smoke at a later age, black smokers bear a significant proportion of tobacco-related cancers with higher rates of oral, lung and oesophageal cancers. ${ }^{12-14}$

Ongoing public health efforts are responsible for the decline in tobacco use; however, significant sociodemographic disparities exist among those who quit smoking. For example, black smokers have a higher number of quit attempts, yet are less successful at quitting. ${ }^{15}$ While higher income and education are known determinants of quitting smoking, ${ }^{16}{ }^{17}$ there are limited data that evaluate social, psychosocial, economic and behavioural determinants of smoking status, especially in black smokers, and in the south, where smoking is prevalent. Understanding facilitators and barriers to a change in smoking status is an essential step to reducing tobacco-related disparities. We addressed this gap in knowledge by using the Reasons for Geographic and Racial Differences in Stroke (REGARDS) study. ${ }^{18}$ The REGARDS study collected in-depth information regarding social, psychosocial, economic and behavioural determinants that may impact one's ability to quit smoking. This national cohort oversampled black smokers and individuals living in the stroke belt of the southeastern USA (North Carolina, South Carolina, Georgia, Tennessee, Mississippi, Alabama, Louisiana and Arkansas ${ }^{19}$; thus, it is an ideal setting to examine sociodemographic determinants of former-smoker status. We evaluated demographic, social, psychosocial, economic and behavioural factors associated with former-smoker status among ever-smoker participants in the REGARDS study. We hypothesised that former smokers would have higher income, higher education, stronger social support, better access to healthcare, healthier diet and less stress, alcohol and television use. We anticipated that these individual determinants would vary by race.

\section{METHODS}

\section{Study design and cohort}

We performed a cross-sectional population-based analysis of participants in the REGARDS cohort. The REGARDS study began in 2003 to investigate the causes for excess stroke mortality among black smokers and people living in the southeastern USA. The cohort consists of 30239 participants from across the continental USA. Participants were aged 45 years or older at baseline; $55 \%$ of the participants resided in the stroke belt, $42 \%$ were black individuals and 55\% were women. Between 2003 and 2007, participants were recruited by mail and then were contacted via telephone for an in-depth health information interview, followed by an in-home exam that involved blood pressure measurement, blood and urine samples, an ECG and medication information. Further details regarding the study design have been previously published. ${ }^{18}$

\section{Patient and public involvement}

Patients were not involved in the design of the current study.

\section{Outcome of interest}

Study participants reported smoking history as packs smoked per day $\times$ total years smoked, age at initiation and current smoking status. The primary outcome was smoking status defined by self-reported 'former-smoker' or 'current-smoker' status in persons who had smoked at least 100 cigarettes in their lifetime.

\section{Definition of covariates}

Specific covariates chosen to be included in our model were those available in the REGARDS database that impacted smoking status based on various levels of influence, including personal, interpersonal, community and environmental levels. Demographic characteristics in the analysis included age at study enrolment, sex, race (self-identified as non-Hispanic white or non-Hispanic black individuals), size of household (number of people), geographical region (defined by the U.S. Census Bureau ${ }^{20}$ as midwest, northeast, south and west) and living environment (urban: $>75 \%$ urban, rural: $<25 \%$ urban or mixed: $25 \%-75 \%$ urban; based on census definition). Economic variables included level of education (less than high school, high school graduate, some college, and college graduate or higher education), annual household income $(<\$ 20000, \$ 20000-\$ 34000, \$ 35000-\$ 74000, \geq \$ 75000$ and not reported) and health insurance (yes vs no). Biological variables (height and weight to calculate body mass index (BMI)) and comorbidities consisting of coronary artery disease, stroke, atrial fibrillation, kidney disease, dyslipidaemia and hypertension were included. Stroke and hypertension were self-reported. Dyslipidaemia was determined by self-reported use of lipid-lowering medication. Coronary artery disease was determined by self-report or by history of myocardial infarction, stenting or bypass surgery. Atrial fibrillation was determined by ECG or by self-report. Kidney disease was determined by a glomerular filtration rate of $<60 \mathrm{~mL} / \mathrm{min}$. Other self-reported measures of health included general health, as well as the mental and physical composite scores of the 12-Item Short-Form Survey (SF-12). ${ }^{21}$ Stress level was determined by Cohen's Perceived Stress Index. ${ }^{22}$ Depressive symptoms were assessed by the Center for Epidemiologic Studies depression scale. ${ }^{23}$

Social variables included marital status (married, single, divorced, widowed or other), number of close 
friends $(<2,2-3,4-5, \geq 6$, based on quartiles of the distribution), number of close relatives $(<2,2-4,5-8,>8$, based on quartiles of the distribution) and percentage of close friends/relatives seen per month. Behavioural characteristics included those that are reflective of a healthy or health-conscious lifestyle. These included physical activity ('How many times per week do you engage in intense physical activity, enough to work up a sweat?' $<3$ vs $\geq 3$ times/week), ${ }^{24}$ medication adherence (assessed by a 4 -item validated scale and classified as low, medium or high), ${ }^{25}$ alcohol use per week (none, moderate or heavy), ${ }^{26}$ adherence to a Mediterranean diet (assessed with the Mediterranean diet score by a three-level categorization as low, medium or high ${ }^{27}$ ) and television use (average number of hours spent watching television every day: $\leq 1,2,3$ and $\geq 4$ ).

\section{Statistical analysis}

We included all REGARDS participants who reported smoking at least 100 cigarettes in their lifetime. Characteristics of participants who reported former-smoker status versus those who reported current-smoker status were compared using $\chi^{2}$ tests of association or t-tests, as appropriate. We built a multivariable logistic regression model and evaluated demographic, social, economic and behavioural determinants associated with formersmoker status in a single model. We excluded anyone with missing values for any of the covariates. Because of the large number of missing values due to Food Frequency Questionnaires that were not returned, we did a sensitivity analysis excluding variables that were derived from that form (television use and Mediterranean diet). The magnitude of association was described as OR with an accompanying 95\% CI. We also examined interactions between race and each of the risk factors using multiplicative interaction terms, with each interaction examined in a separate model. We used SAS V.9.4 for all statistical analyses. All hypothesis testing was two sided, and to be conservative due to the exploratory nature of the analyses, we considered $p<0.01$ to be significant.

\section{RESULTS}

Of the 30239 REGARDS participants, 16463 (54.4\%) reported smoking at least 100 cigarettes during their lifetime and were included in this analysis. Of these, $4396(27 \%)$ participants identified as current smokers and $12067(73 \%)$ as former smokers. Table 1 describes the characteristics of the cohort, overall and by current smoking status. The mean age at study enrolment was 64.9 years $(\mathrm{SD}=9.0), 47 \%$ were women, and $41 \%$ were black individuals. The majority resided in the south $(67 \%)$ and in urban areas $(72 \%)$ and were married $(59 \%)$. With respect to health comorbidities, a majority reported having a history of hypertension $(59 \%)$.

\section{Determinants of former-smoker status}

Demographic factors associated with former-smoker status

Table 2 describes the determinants of former-smoker status. Male sex $(\mathrm{OR}=1.35,95 \%$ CI 1.18 to 1.56$)$, health insured status ( $\mathrm{OR}=1.39,95 \% \mathrm{CI} 1.10$ to 1.75 ), higher education and higher income were associated with increased odds of reporting former-smoker status, such that college graduates or those with higher education $(\mathrm{OR}=1.39,95 \% \mathrm{CI} 1.10$ to 1.77$)$ and an annual income of \$35 000-\$74000 (OR=1.31, 95\% CI 1.07 to 1.61 ) had the highest odds of former-smoker status relative to those with less than high school education and those with $<\$ 20000$ income, respectively. The odds of former-smoker status were lower among black smokers than white smokers (OR=0.62, 95\% CI 0.53 to 0.72), among younger adults (45-64 years) compared with older adults $(\geq 65$ years; $\mathrm{OR}=0.34,95 \% \mathrm{CI} 0.29$ to 0.39 ) and among those with larger household sizes compared with smaller household sizes (OR=0.90, 95\% CI 0.86 to 0.095$)$. Geographical region and living environment were not associated with smoking status.

\section{Social factors associated with former-smoker status}

Marital status was significantly associated with reporting former-smoker status with single $(\mathrm{OR}=0.66,95 \%$ CI 0.51 to 0.87 ), divorced ( $\mathrm{OR}=0.60,95 \% \mathrm{CI} 0.50$ to 0.72$)$ and widowed $(\mathrm{OR}=0.70,95 \% \mathrm{CI} 0.57$ to 0.85$)$ participants having lower odds of former-smoker status, compared with those who were married. However, the number of close friends or close relatives, and the frequency of social interactions with friends/relatives were not associated with former-smoker status in the full model.

\section{Comorbidities associated with former-smoker status}

History of atrial fibrillation ( $\mathrm{OR}=1.42,95 \%$ CI 1.13 to 1.79), chronic kidney disease ( $\mathrm{OR}=1.38,95 \%$ CI 1.10 to 1.73 ) and hypertension ( $\mathrm{OR}=1.24,95 \% \mathrm{CI}, 1.08$ to 1.42$)$ were associated with higher odds of reporting formersmoker status, while other comorbidities were not, nor was physical health as summarised by the SF-12 measure. Those with BMI in the non-obese categories and those reporting their general health as less than excellent had significantly lower odds of former-smoker status, compared with those with obesity and those with excellent general health, respectively. Perceived stress and depressive symptoms were not associated with former-smoker status. However, higher mental health summary score by the SF-12 was associated with former-smoker status (1 SD change: $\mathrm{OR}=1.13,95 \% \mathrm{CI} 1.04$ to 1.23$)$.

\section{Behavioural factors associated with former-smoker status}

Physical activity performed three times or more per week (compared with physical activity $<3 \times$ per week: $\mathrm{OR}=1.26$, 95\% CI 1.11 to 1.43 ) and high adherence to a Mediterranean diet (compared with low adherence to a Mediterranean diet: $\mathrm{OR}=2.20,95 \%$ CI 1.84 to 2.64 ) were associated with higher odds of reporting former-smoker status. Participants who did not consume alcohol on a 
Open access

Table 1 Baseline characteristics of former and current smokers

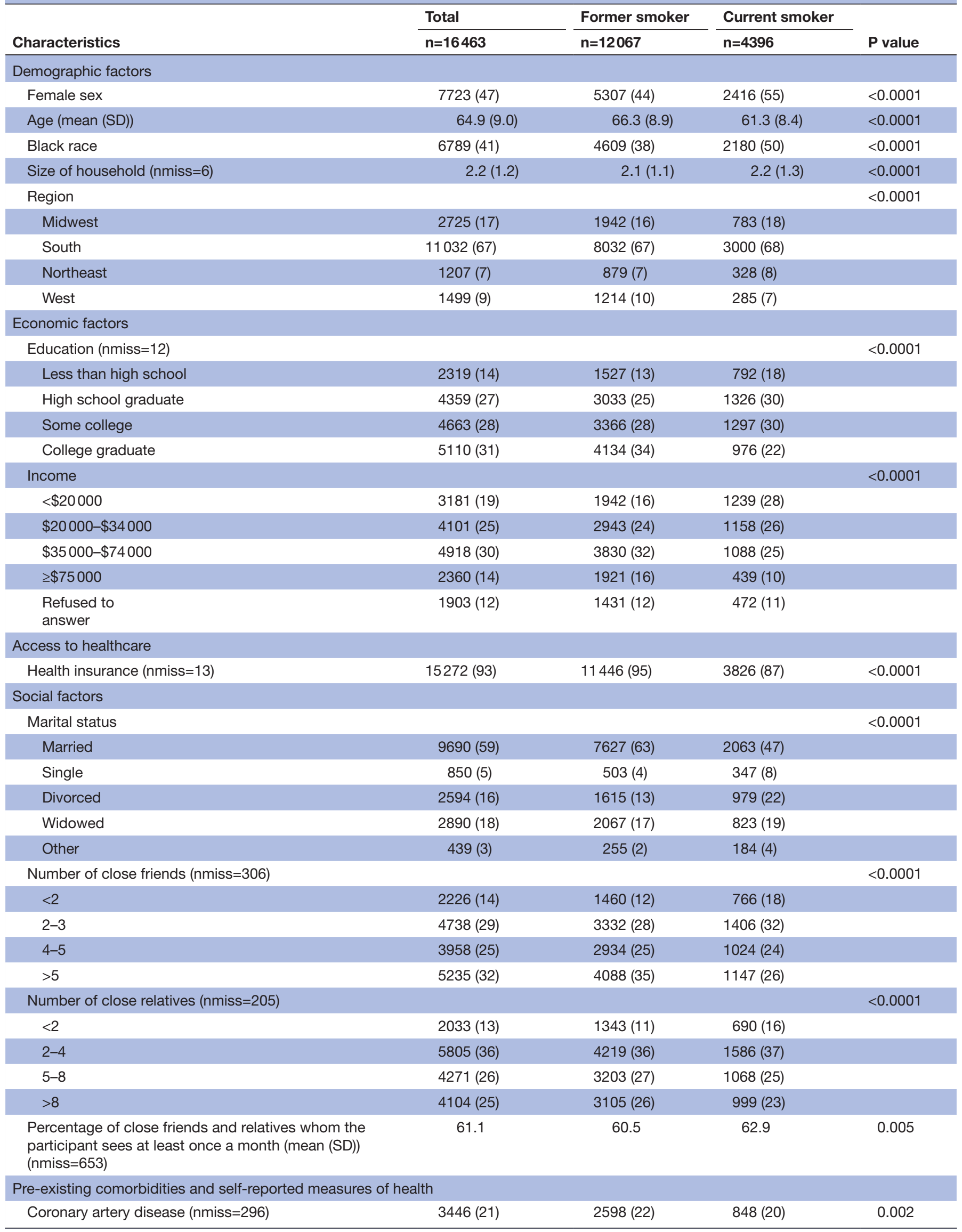

Continued 
Table 1 Continued

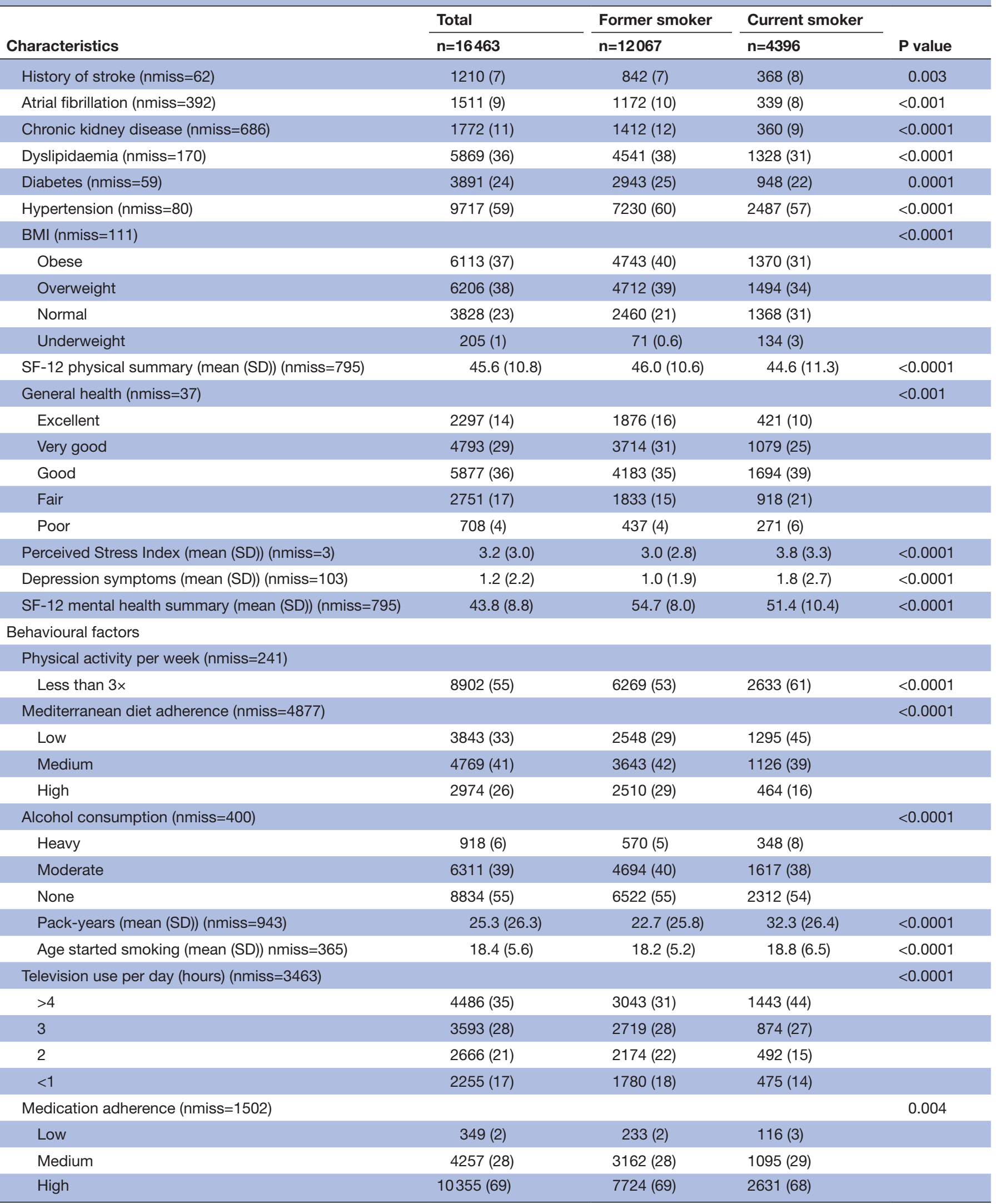

BMI, body mass index; nmiss, number missing; SF-12, 12-Item Short-Form Survey. 
Table 2 Multivariable analysis of factors associated with former-smoker status $(n=7322)$

\begin{tabular}{|c|c|c|}
\hline Variable & OR $(95 \% \mathrm{Cl})$ & $P$ value \\
\hline \multicolumn{3}{|l|}{ Demographic factors } \\
\hline \multicolumn{3}{|l|}{ Age (years) } \\
\hline $\begin{array}{l}65+ \\
45-64\end{array}$ & $\begin{array}{l}\text { REF } \\
0.34 \text { (0.29 to } 0.39)\end{array}$ & $<0.001$ \\
\hline Sex & & $<0.001$ \\
\hline Female & REF & \\
\hline Male & 1.35 (1.18 to 1.56$)$ & \\
\hline Race & & $<0.001$ \\
\hline White & REF & \\
\hline Black & 0.62 (0.53 to 0.72 ) & \\
\hline Size of household & 0.90 (0.86 to 0.95$)$ & $<0.001$ \\
\hline Region & & 0.5 \\
\hline West & REF & \\
\hline Northeast & 0.87 (0.64 to 1.19 ) & \\
\hline Midwest & 0.82 (0.63 to 1.06$)$ & \\
\hline South & 0.85 (0.67 to 1.07$)$ & \\
\hline Living environment & & 0.4 \\
\hline Urban & REF & \\
\hline Rural & 1.03 (0.84 to 1.26$)$ & \\
\hline Mixed & 0.88 (0.73 to 1.07$)$ & \\
\hline
\end{tabular}

\section{Economic factors}

Education

0.01

Less than high school REF

High school graduate

Some college

$1.10(0.88$ to 1.36$)$

College graduate

1.12 (0.90 to 1.39$)$

1.39 (1.10 to 1.77$)$

\begin{tabular}{|c|c|c|}
\hline Income & & 0.003 \\
\hline$<\$ 20000$ & REF & \\
\hline$\$ 20000-\$ 34000$ & $1.16(0.96$ to 1.40$)$ & \\
\hline$\$ 35000-\$ 74000$ & 1.31 (1.07 to 1.61$)$ & \\
\hline$\geq \$ 75000$ & 1.27 (0.98 to 1.64$)$ & \\
\hline Refused to answer & 1.69 (1.29 to 2.20$)$ & \\
\hline \multicolumn{3}{|l|}{ Access to healthcare } \\
\hline Health insurance & 1.39 (1.10 to 1.75$)$ & 0.007 \\
\hline \multicolumn{3}{|l|}{ Social factors } \\
\hline Marital status & & $<0.001$ \\
\hline Married & REF & \\
\hline Single & 0.66 (0.51 to 0.87$)$ & \\
\hline Divorced & 0.60 (0.50 to 0.72$)$ & \\
\hline Widowed & 0.70 (0.57 to 0.85$)$ & \\
\hline Other & 0.78 (0.52 to 1.17$)$ & \\
\hline
\end{tabular}

Continued

\begin{tabular}{|c|c|c|}
\hline Variable & OR $(95 \% \mathrm{Cl})$ & $P$ value \\
\hline Number of close friends & & 0.4 \\
\hline$<2$ & REF & \\
\hline $2-3$ & $1.03(0.84$ to 1.23$)$ & \\
\hline $4-5$ & $0.91(0.74$ to 1.13$)$ & \\
\hline$>5$ & $0.92(0.74$ to 1.14$)$ & \\
\hline Number of close relatives & & 0.2 \\
\hline$<2$ & REF & \\
\hline $2-4$ & 1.21 (0.99 to 1.48$)$ & \\
\hline $5-8$ & $1.24(1.00$ to 1.54$)$ & \\
\hline$>8$ & $1.21(0.96$ to 1.51$)$ & \\
\hline $\begin{array}{l}\text { Percentage of close } \\
\text { friends and relatives whom } \\
\text { the participant sees at } \\
\text { least once a month }\end{array}$ & 0.97 (0.92 to 1.03$)$ & 0.4 \\
\hline \multicolumn{3}{|c|}{ Pre-existing comorbidities and self-reported measures of health } \\
\hline Coronary artery disease & 0.95 (0.80 to 1.12$)$ & 0.5 \\
\hline History of stroke & $0.99(0.77$ to 1.28$)$ & 0.9 \\
\hline Atrial fibrillation & $1.42(1.13$ to 1.79$)$ & 0.002 \\
\hline Chronic kidney disease & $1.38(1.10$ to 1.73$)$ & 0.005 \\
\hline Dyslipidaemia & 1.05 (0.92 to 1.12$)$ & 0.5 \\
\hline Diabetes & $1.17(1.00$ to 1.38$)$ & 0.05 \\
\hline Hypertension & 1.24 (1.08 to 1.42$)$ & 0.002 \\
\hline \multicolumn{3}{|l|}{ BMI } \\
\hline $\begin{array}{l}\text { Obese } \\
\text { Overweight } \\
\text { Normal } \\
\text { Underweight }\end{array}$ & $\begin{array}{l}\text { REF } \\
0.62(0.54 \text { to } 0.72) \\
0.37(0.31 \text { to } 0.44) \\
0.17(0.10 \text { to } 0.28)\end{array}$ & $<0.001$ \\
\hline $\begin{array}{l}\text { SF-12 physical summary } \\
\text { (1 SD change) }\end{array}$ & 0.97 (0.89 to 1.06$)$ & 0.5 \\
\hline General health & & $<0.001$ \\
\hline Excellent & REF & \\
\hline Very good & 0.68 (0.54 to 0.84$)$ & \\
\hline Good & 0.49 (0.39 to 0.61$)$ & \\
\hline Fair & 0.56 (0.42 to 0.75$)$ & \\
\hline Poor & 0.63 (0.40 to 0.97$)$ & \\
\hline $\begin{array}{l}\text { Perceived Stress Index (1 } \\
\text { SD change) }\end{array}$ & 0.94 (0.87 to 1.01$)$ & 0.07 \\
\hline $\begin{array}{l}\text { Depression symptoms ( } 1 \\
\text { SD change) }\end{array}$ & 0.99 (0.91 to 1.07$)$ & 0.7 \\
\hline $\begin{array}{l}\text { SF-12 mental health } \\
\text { summary (1 SD change) }\end{array}$ & $1.13(1.04$ to 1.23$)$ & 0.005 \\
\hline \multicolumn{3}{|l|}{ Behavioural factors } \\
\hline Physical activity per week & & $<0.001$ \\
\hline Less than $3 x$ & REF & \\
\hline $3 \times$ or more & $1.26(1.11$ to 1.43$)$ & \\
\hline
\end{tabular}

Continued 


\begin{tabular}{|c|c|c|}
\hline Variable & OR (95\% Cl) & $P$ value \\
\hline $\begin{array}{l}\text { Mediterranean diet } \\
\text { adherence }\end{array}$ & & $<0.001$ \\
\hline Low & REF & \\
\hline Medium & 1.46 (1.27 to 1.67$)$ & \\
\hline High & 2.20 (1.84 to 2.64$)$ & \\
\hline Alcohol consumption & & $<0.001$ \\
\hline Heavy & REF & \\
\hline Moderate & 1.15 (0.90 to 1.46$)$ & \\
\hline None & 1.50 (1.18 to 1.91$)$ & \\
\hline \multicolumn{3}{|l|}{ Cigarette smoking } \\
\hline $\begin{array}{l}\text { Pack-years (one pack } \\
\text { change) }\end{array}$ & 0.98 (0.98 to 0.98$)$ & $<0.001$ \\
\hline \multicolumn{3}{|l|}{ Cigarette smoking } \\
\hline $\begin{array}{l}\text { Age started smoking } \\
\text { (1-year change) }\end{array}$ & 0.96 (0.95 to 0.97$)$ & $<0.001$ \\
\hline $\begin{array}{l}\text { Television use per } \\
\text { day (hours) }\end{array}$ & & $<0.001$ \\
\hline$>4$ & REF & \\
\hline 3 & 1.13 (0.97 to 1.32$)$ & \\
\hline 2 & 1.45 (1.21 to 1.74$)$ & \\
\hline$<1$ & 1.32 (1.10 to 1.60$)$ & \\
\hline Medication adherence & & 0.05 \\
\hline Low & REF & \\
\hline Medium & 1.05 (0.69 to 1.60$)$ & \\
\hline High & 0.89 (0.59 to 1.34$)$ & \\
\hline
\end{tabular}

BMI, body mass index; REF, reference; SF-12, 12-Item ShortForm Survey.

weekly basis had higher odds of former-smoker status (compared with heavy alcohol use weekly: $\mathrm{OR}=1.50$, $95 \%$ CI 1.18 to 1.91). Higher number of pack-years and older age of starting smoking were associated with lower odds of former-smoker status. Participants who watched 2 hours or less of television per day had higher odds of reporting former-smoker status (compared with $\geq 4$ hours: 2 hours: $\mathrm{OR}=1.45,95 \%$ CI 1.21 to 1.74 ; <1 hour: $\mathrm{OR}=1.32$, $95 \%$ CI 1.10 to 1.60$)$.
Sensitivity analyses

Excluding variables derived from the Food Frequency Questionnaire deemed the results of the analysis largely unchanged (online supplementary table 1). The exceptions were that now the northeast, midwest and south all had significant lower odds of former-smoker status than the west, while participants with diabetes and dyslipidaemia each had higher odds of former-smoker status than participants without diabetes and dyslipidaemia.

Interaction between key determinants and race

We present findings for the significant interactions between race and determinants of former-smoker status in table 3. We found a significant interaction $(p=0.0002)$ between race and alcohol consumption, such that black participants had significantly higher odds of reporting former-smoker status if they were abstinent from alcohol $(\mathrm{OR}=2.32,95 \% \mathrm{CI} 1.47$ to 3.68$)$ compared with those participants with heavy alcohol use; however, the relationship was not statistically significant in white participants. Self-reported history of dyslipidaemia and race interacted such that black participants with a history of dyslipidaemia had higher odds of former-smoker status $(\mathrm{OR}=1.31,95 \%$ CI 1.06 to 1.62); however, the relationship was not statistically significant in white participants.

\section{CONCLUSION}

We evaluated predictors of former-smoker status in the REGARDS cohort and found that male sex, white race, income (\$35000-\$74000) and higher education were associated with higher odds of reporting formersmoker status. Out of the potentially modifiable social and behavioural factors that were used in our model, all behavioural factors were significantly associated with smoking status, including television use, alcohol use, exercise and adherence to a Mediterranean diet. With the exception of marriage, no social factors were associated with smoking status. In addition, stress level and depression, which have been previously associated with smoking, ${ }^{28} 29$ had no association with smoking status in our study. In our interaction analysis, we found that those who abstained from alcohol, compared with those who heavily used alcohol, and those who had a history

Table 3 Interactions between race and key determinants associated with former smoker status $(n=7322)$

\begin{tabular}{|c|c|c|c|}
\hline & White race & Black race & Interaction \\
\hline Variable & OR (95\% Cl) & OR (95\% Cl) & $P$ value \\
\hline Dyslipidaemia & 0.92 (0.78 to 1.08$)$ & 1.31 (1.06 to 1.62$)$ & 0.008 \\
\hline Heavy & REF & REF & \\
\hline Moderate & $1.12(0.84$ to 1.50$)$ & 1.30 (0.81 to 2.09$)$ & \\
\hline
\end{tabular}

REF, reference. 
of dyslipidaemia improved the odds of reporting formersmoker status among black, but not white, participants.

Addiction to tobacco and alcohol coexist as positive reinforcements for each other, with alcohol consumption increasing the urge to smoke $\mathrm{e}^{30}$ and nicotine inhalation increasing the urge to drink. ${ }^{31}$ Tobacco companies are well aware of this association and have tailored marketing strategies to encourage the concurrent use of both substances. ${ }^{32}$ Tobacco and alcohol use disorders commonly present together with significant negative consequences occurring from the abuse of both substances. Former smokers are four times more likely to experience initial smoking relapse while drinking. ${ }^{33}$ Alcoholics smoke more cigarettes per day and score higher on nicotine dependence measures compared with other drug abusers. ${ }^{34}$ In addition, alcohol abuse places smokers at higher risk of certain cancers, including oral, throat and oesophageal cancers. ${ }^{35}$

While alcohol use is known to increase tobacco use, ${ }^{36}$ the magnitude of association between abstaining from alcohol and quitting smoking in black populations has implications for future interventions. Black and white populations have cultural and social differences that warrant consideration when designing and evaluating cessation interventions. Racial minorities are historically under-represented in intervention trials, ${ }^{37}$ and while evidence-based guidelines may recommend specific interventions for smoking cessation, population-based interventions are most effective when designed to reach all populations equally. A secondary analysis of the Timing of Alcohol and Smoking Cessation study evaluated the racial/ethnic differences in outcomes of 499 tobacco and alcohol abuse participants undergoing cessation treatments, comparing those who had concurrent alcohol abstinence and smoking cessation treatment versus those who had alcohol abstinence treatment followed by a smoking intervention 6 months later. Alcohol abstinence outcomes (reduced time to first use of alcohol after treatment and risk of alcohol resumption) were worse for white participants in the concurrent group compared with the delayed group, but were not significantly different between black groups, ${ }^{38}{ }^{39}$ suggesting that the optimal approach to concurrent cessation therapy differs by race/ ethnicity. Previous interventions have treated different types of substance abuse separately, and while some data support treating both conditions simultaneously, further studies are needed to define best practices for treating concomitant tobacco and alcohol abuses, ${ }^{40}$ specifically in racial/ethnic minority populations.

Our study also reports an interaction between race and dyslipidaemia. Black smokers suffer disproportionately from cardiovascular diseases ${ }^{41}$ and treatment of risk factors, including dyslipidaemia, is vital to protect against cardiovascular events. However, black smokers with dyslipidaemia are less likely to be aware of their disease and less likely to be treated for it. ${ }^{42}$ The presence of dyslipidaemia in our study was defined by self-reported use of lipid-lowering medication and was associated with higher odds of former-smoker status in black individuals. This suggests that the awareness of a dyslipidaemia diagnosis could have an impact on smoking status, which has been previously reported. ${ }^{43}$

Our report adds to existing literature documenting determinants of smoking status. Yang et al evaluated determinants of smoking cessation in a Korean population and found results similar to our study; married status, higher education, alcohol abstinence and disease morbidity were associated with quitting smoking. ${ }^{44}$ Data from the US National Health and Wellness survey found that Hispanic ethnicity (vs non-Hispanic white individuals), higher income, obese weight, regular physical activity, insured status, and those who received the influenza vaccine and who were taking steps to lose weight were more likely to be former smokers. ${ }^{45}$ A systematic review including prospective studies analysing predictors of successful smoking cessation found that lower levels of cigarette dependence predicted smoking cessation. ${ }^{46}$ Few studies have examined whether social, behavioural and economic determinants of smoking cessation vary by sociodemographic subgroups, particularly race, although these findings are desirable for targeted intervention development. In a report from the National Epidemiological Study of Alcohol and Related Conditions evaluating determinants of smoking cessation, Agrawal et al stratified results by age and found that alcoholism was associated with persistent smoking in older adults, but not in younger adults. ${ }^{17}$ In a study evaluating racial differences in attempts to quit smoking and smoking cessation after screening for lung cancer, black smokers had more frequent 24-hour and 7-day quit attempts; however, the 6-month cessation rate between black and white smokers did not differ. ${ }^{47}$ This further highlights the need for identifying factors that may help promote successful quitting in black smokers. The strength of our study lies in a deeper exploration of the unique interaction of determinants of smoking status by key demographic characteristics. Using a resource that is distinctively enriched to identify racial differences, we make a novel observation that race interacts with alcohol use and history of dyslipidaemia in determining former smoking status.

The results of our study should be interpreted in light of several limitations. Former-smoker status was self-reported and not defined by biochemical confirmation; therefore, we can only assess status as reported by the participant. However, in large population-based studies, biochemical confirmation can lead to selection bias unrelated to smoking status. ${ }^{48}$ Our study examined smoking status at time of study entry, and therefore the determinants of each participant were reflective of study entry and not necessarily at the time of smoking cessation. In addition, factors associated with former-smoker status may also reflect differences in those who initiate smoking (men vs women). By design, REGARDS enrolled only black and white participants who were 45 years or older; therefore, it could not address smoking status of those who were younger or were from other racial/ethnic 
backgrounds. Our study highlights the importance of race in tobacco-related disparities; however, race in the current study reflects a social construct and does not imply ancestry as participants self-identified as 'black' or 'white'. Finally, we performed a cross-sectional analysis, and therefore the data presented do not establish causation. For example, factors such as improved diet and increased physical activity may be co-occurring changes rather than determinants of smoking status changes. However, the associations and interactions uncovered in our study call for further complex investigations that may establish causality.

Despite the progress being made in reducing tobacco use, there has been no consistent increase in cessation rates $^{49}$ and fewer than 1 in 10 smokers who wish to quit are able to quit successfully. ${ }^{15}$ Smokers who are black have a higher number of quit attempts yet are less successful at quitting. ${ }^{50}$ In an environment that impedes quitting smoking, we are further petitioned to address factors that are within our reach, including alcohol abstinence and dyslipidaemia education and treatment, especially in black smokers. It is critically important that alcohol and tobacco use intervention studies ensure diverse recruitment and analyse results by race in order to assess the effectiveness of interventions in minority groups. The results of our study can inform public health professionals and clinicians to consider including alcohol abstinence and dyslipidaemia education as key elements of a culturally sensitive intervention to achieve smoking cessation in black smokers.

\section{Author affiliations}

${ }^{1}$ Pulmonary and Critical Care Medicine, University of Alabama at Birmingham, Birmingham, Alabama, USA

${ }^{2}$ Department of Epidemiology and Biostatistics, Drexel University, Philadelphia, Pennsylvania, USA

${ }^{3}$ Department of Epidemiology, University of Alabama at Birmingham, Birmingham, Alabama, USA

${ }^{4}$ Department of Medicine, University of Vermont College of Medicine, Burlington, Vermont, USA

${ }^{5}$ Department of Public Health Services, Medical University of South Carolina, College of Medicine, Charleston, South Carolina, USA

${ }^{6}$ Department of Preventive Medicine, University of Alabama at Birmingham, Birmingham, Alabama, USA

${ }^{7}$ Institute for Cancer Outcomes and Survivorship, University of Alabama at Birmingham, Birmingham, Alabama, USA

Contributors TMP and SB: study design, data analysis, data interpretation, drafting of the manuscript, editing of the manuscript, final approval and agreement for accountability. CW and LAM: study design, data analysis, data interpretation, editing of the manuscript, final approval and agreement for accountability. VJH, MC, AMM, KFH, ALC and MTD: study design, data interpretation, editing of the manuscript, final approval and agreement for accountability.

Funding TMP is supported by the National Institutes of Health grant 5T32HL105346-07. This research project is supported by a cooperative agreement, U01 NS041588, from the National Institute of Neurological Disorders and Stroke, the National Institutes of Health and the Department of Health and Human Service. The content is solely the responsibility of the authors and does not necessarily represent the official views of the National Institute of Neurological Disorders and Stroke or the National Institutes of Health. Representatives of the funding agency have been involved in the review of the manuscript but were not directly involved in the collection, management, analysis or interpretation of the data. The authors thank the other investigators, the staff and the participants of the Reasons for
Geographic and Racial Differences in Stroke (REGARDS) study for their valuable contributions (a full list of participating REGARDS investigators and institutions can be found at http://www.regardsstudy.org). This work is not under consideration for publication elsewhere. Some contents were presented as an abstract at the American Thoracic Society Conference in May 2018.

Competing interests TMP, CW, LAM, VJH, MC, AMM and KFH have no conflicts of interests to report. ALC reports research support from Boehringer Ingelheim and consulting fees from AstraZeneca and Novo Nordisk. MTD reports grants from the National Institutes of Health involving the work under consideration for publication; grants from the Department of Defense outside the submitted work; contracted clinical trial support from AstraZeneca, Boehringer Ingeheim, Boston Scientific, GlaxoSmithKline, Novartis, PneumRxBTG, Pulmonx and Yungjin outside the submitted work; and consulting fees from AstraZeneca, GlaxoSmithKline and PneumRxBTG outside the submitted work. SB has no conflicts to report.

Patient consent for publication Not required.

Ethics approval The institutional review boards of participating institutions approved the study methods. The ethics approval for the initial study allowed for secondary analyses without additional ethics review.

Provenance and peer review Not commissioned; externally peer reviewed.

Data sharing statement This study uses data from the Reasons for Geographic and Racial Differences in Stroke (REGARDS) cohort. In order to abide by its obligations with the National Institutes of Health/National Institute of Neurological Disorders and Stroke and the Institutional Review Board of the University of Alabama at Birmingham, REGARDS facilitates data sharing through formal data use agreements. Any investigator is welcome to access the REGARDS data through this process. Requests for data access may be sent to regardsadmin@uab.edu.

Open access This is an open access article distributed in accordance with the Creative Commons Attribution Non Commercial (CC BY-NC 4.0) license, which permits others to distribute, remix, adapt, build upon this work non-commercially, and license their derivative works on different terms, provided the original work is properly cited, appropriate credit is given, any changes made indicated, and the use is non-commercial. See: http://creativecommons.org/licenses/by-nc/4.0/.

\section{REFERENCES}

1. Health CO on S and. Smoking and Tobacco Use; Fact Sheet; Adult Cigarette Smoking in the United States; [Internet]. Smoking and Tobacco Use. http://www.cdc.gov/tobacco/data_statistics/fact sheets/adult_data/cig_smoking/ (cited 28 Nov 2016).

2. Health $\mathrm{CO}$ on $\mathrm{S}$ and. Smoking and Tobacco Use; Fact Sheet; Health Effects of Cigarette Smoking [Internet]. Smoking and Tobacco Use. http://www.cdc.gov/tobacco/data_statistics/fact_sheets/health_ effects/effects_cig_smoking/(cited 28 Nov 2016).

3. Health NC for CDP and HP (US) O on S and. Introduction, Summary, and Conclusions [Internet]. Centers for Disease Control and Prevention. 2014 https://www.ncbi.nlm.nih.gov/books/NBK294320/ (cited 29 Nov 2016).

4. Tobacco Use Among U.S. Racial/ethnic minority groups - complete report.pdf [Internet]. https://www.cdc.gov/tobacco/data_statistics/ sgr/1998/complete_report/pdfs/complete_report.pdf (cited 27 Apr 2018).

5. National Center for Chronic Disease Prevention and Health Promotion (US) Office on Smoking and Health. The Health Consequences of Smoking - 50 Years of Progress: A Report of the Surgeon General [Internet. Atlanta (GA: Centers for Disease Control and Prevention (US). http://www.ncbi.nlm.nih.gov/books/ NBK179276/

6. Loop MS, Howard G, de Los Campos G, et al. Heat maps of hypertension, diabetes mellitus, and smoking in the continental United States. Circ Cardiovasc Qual Outcomes 2017;10.

7. Pérez-Stable EJ, Herrera B, Jacob P, et al. Nicotine metabolism and intake in black and white smokers. JAMA 1998;280:152-6.

8. Income, Poverty, and Health Insurance Coverage in the United States: 2009 - p60-238.pdf. https://www.census.gov/prod/ 2010pubs/p60-238.pdf

9. Trinidad DR, Pérez-Stable EJ, White MM, et al. A nationwide analysis of US racial/ethnic disparities in smoking behaviors, smoking cessation, and cessation-related factors. Am J Public Health 2011;101:699-706.

10. Moreland-Russell S, Harris J, Snider D, et al. Disparities and menthol marketing: additional evidence in support of point of sale policies. Int J Environ Res Public Health 2013;10:4571-83. 
11. Alexander LA, Trinidad DR, Sakuma K-LK, et al. Why We Must Continue to Investigate Menthol's Role in the African American Smoking Paradox. Nicotine \& Tobacco Research 2016;18(suppl 1):S91-S101.

12. Shiboski CH, Schmidt BL, Jordan RC. Racial disparity in stage at diagnosis and survival among adults with oral cancer in the US. Community Dent Oral Epidemiol 2007;35:233-40.

13. Baquet CR, Commiskey P, Mack K, et al. Esophageal cancer epidemiology in blacks and whites: racial and gender disparities in incidence, mortality, survival rates and histology. J Natl Med Assoc 2005;97:8.

14. Park ER, Japuntich SJ, Traeger L, et al. Disparities between blacks and whites in tobacco and lung cancer treatment. Oncologist 2011;16:1428-34.

15. Babb S, Malarcher A, Schauer G, et al. Quitting smoking among adults - United States, 2000-2015. MMWR Morb Mortal Wkly Rep 2017;65:1457-64.

16. Reid JL, Hammond D, Boudreau C, et al. Socioeconomic disparities in quit intentions, quit attempts, and smoking abstinence among smokers in four western countries: findings from the International Tobacco Control Four Country Survey. Nicotine Tob Res 2010;12(suppl 1):S20-S33.

17. Agrawal A, Sartor C, Pergadia ML, et al. Correlates of smoking cessation in a nationally representative sample of U.S. adults. Addict Behav 2008;33:1223-6.

18. Howard VJ, Cushman M, Pulley L, et al. The reasons for geographic and racial differences in stroke study: objectives and design. Neuroepidemiology 2005;25:135-43.

19. Lanska DJ, Kuller LH. The geography of stroke mortality in the United States and the concept of a stroke belt. Stroke 1995;26:1145-9.

20. Geography UCB. Geographic Terms and Concepts - Census Divisions and Census Regions [Internet]. 2010 https://www.census. gov/geo/reference/gtc/gtc_census_divreg.html (cited 2017 Aug 16).

21. Ware J, Kosinski M, Keller SD. A 12-Item short-form health survey: construction of scales and preliminary tests of reliability and validity. Med Care 1996;34:220-33.

22. Cohen S, Kamarck T, Mermelstein R. A global measure of perceived stress. J Health Soc Behav 1983;24:385-96.

23. Orme JG, Reis J, Herz EJ. Factorial and discriminant validity of the Center for Epidemiological Studies Depression (CES-D) scale. J Clin Psychol 1986;42:28-33.

24. Washburn RA, Goldfield SR, Smith KW, et al. The validity of selfreported exercise-induced sweating as a measure of physical activity. Am J Epidemiol 1990;132:107-13.

25. Morisky DE, Green LW, Levine DM. Concurrent and predictive validity of a self-reported measure of medication adherence. Med Care 1986;24:67-74.

26. Cunningham SA, Mosher A, Judd SE, et al. Alcohol Consumption and Incident Stroke Among Older Adults. J Gerontol Ser B https:// academic.oup.com/psychsocgerontology/advance-article/doi/ (cited 26 Mar 2018).

27. Mantzoros CS, Williams CJ, Manson JE, et al. Adherence to the Mediterranean dietary pattern is positively associated with plasma adiponectin concentrations in diabetic women. Am J Clin Nutr 2006;84:328-35.

28. Luger TM, Suls J, Vander Weg MW. How robust is the association between smoking and depression in adults? A meta-analysis using linear mixed-effects models. Addict Behav 2014;39:1418-29.

29. Lawless MH, Harrison KA, Grandits GA, et al. Perceived stress and smoking-related behaviors and symptomatology in male and female smokers. Addict Behav 2015;51:80-3.
30. King AC, Epstein AM. Alcohol dose-dependent increases in smoking urge in light smokers. Alcohol Clin Exp Res 2005;29:547-52.

31. Barrett SP, Tichauer M, Leyton M, et al. Nicotine increases alcohol self-administration in non-dependent male smokers. Drug Alcohol Depend 2006;81:197-204.

32. Jiang N, Ling PM. Reinforcement of smoking and drinking: tobacco marketing strategies linked with alcohol in the United States. Am J Public Health 2011;101:1942-54.

33. Kahler CW, Spillane NS, Metrik J. Alcohol use and initial smoking lapses among heavy drinkers in smoking cessation treatment. Nicotine Tob Res 2010;12:781-5.

34. Burling TA, Ziff DC. Tobacco smoking: a comparison between alcohol and drug abuse inpatients. Addict Behav 1988;13:185-90.

35. Johnson N. Tobacco use and oral cancer: a global perspective. $J$ Dent Educ 2001;65:328-39.

36. Hurley LL, Taylor RE, Tizabi Y. Positive and negative effects of alcohol and nicotine and their interactions: a mechanistic review. Neurotox Res 2012;21:57-69.

37. Cox LS, Okuyemi K, Choi WS, et al. A Review of Tobacco Use Treatments in U. S. Ethnic Minority Populations. Am J Health Promot AJHP 2011;25(5 0):S11-30.

38. Joseph AM, Willenbring ML, Nugent SM, et al. A randomized tria of concurrent versus delayed smoking intervention for patients in alcohol dependence treatment. J Stud Alcohol 2004;65:681-91.

39. Fu SS, Kodl M, Willenbring M, et al. Ethnic differences in alcohol treatment outcomes and the effect of concurrent smoking cessation treatment. Drug Alcohol Depend 2008;92:61-8.

40. Kodl M, Fu SS, Joseph AM. Tobacco cessation treatment for alcoholdependent smokers: when is the best time? Alcohol Res Health 2006;29:203-7.

41. Ferdinand KC. Coronary heart disease and lipid-modifying treatment in African American patients. Am Heart J 2004;147:774-82.

42. Nieto FJ, Alonso J, Chambless LE, et al. Population awareness and control of hypertension and hypercholesterolemia. The Atherosclerosis Risk in Communities study. Arch Intern Med 1995; 155:677-84.

43. Kwon JA, Jeon W, Park EC, et al. Effects of Disease Detection on Changes in Smoking Behavior. Yonsei Med J 2015;56:1143-9.

44. Yang JJ, Song M, Yoon HS, et al. What are the major determinants in the success of smoking cessation: results from the health examinees study. PLoS One 2015;10:e0143303.

45. Goren A, Annunziata K, Schnoll RA, et al. Smoking cessation and attempted cessation among adults in the United States. PLoS One 2014;9:e93014

46. Vangeli E, Stapleton J, Smit ES, et al. Predictors of attempts to stop smoking and their success in adult general population samples: a systematic review. Addiction 2011;106:2110-21.

47. Kumar P, Gareen IF, Lathan C, et al. Racial differences in tobacco cessation and treatment usage after lung screening: an examination of the national lung screening trial. Oncologist 2016;21:40-9.

48. SRNT Subcommittee on Biochemical Verification. Biochemical verification of tobacco use and cessation. Nicotine Tob Res Off $J$ Soc Res Nicotine Tob 2002;4:149-59.

49. Zhu SH, Lee M, Zhuang YL, et al. Interventions to increase smoking cessation at the population level: how much progress has been made in the last two decades? Tob Control 2012;21:110-8.

50. Centers for Disease Control and Prevention (CDC). Quitting smoking among adults--United States, 2001-2010. MMWR Morb Mortal Wkly Rep 2011;60:1513-9. 